\title{
Scan-free direct measurement of an one-million-dimensional photonic state
}

\author{
Zhimin Shi $^{1, *}$, Mohammad Mirhosseini' ${ }^{2}$, Jessica Margiewicz $^{1}$, Mehul Malik ${ }^{2,3}$, \\ Freida Rivera ${ }^{1}$, Ziyi Zhu ${ }^{1}$ and Robert W. Boyd ${ }^{2,4}$ \\ ${ }^{1}$ Department of Physics, University of South Florida, Tampa, Florida 33620, USA \\ ${ }^{2}$ The Institute of Optics, University of Rochester, Rochester, New York 14627 USA \\ ${ }^{3}$ Institute for Quantum Optics and Quantum Information (IQOQI), Austrian Academy of Sciences, Boltzmanngasse 3, \\ A-1090 Vienna, Austria \\ ${ }^{4}$ Department of Physics, University of Ottawa, Ottawa, ON K1N 6N5 Canada \\ zhiminshi@usf.edu
}

\begin{abstract}
We describe a technique that directly measures a pure quantum state of arbitrary dimensions with a single setting of the measurement apparatus. We demonstrate our method by measuring a million-dimensional photonic spatial state.
\end{abstract}

(C) 2015 Optical Society of America

OCIS codes: $270.5585,120.0120,120.4640,120.3930$

The state vector of a pure quantum system is a set of complex probability amplitudes used for describing the system in each state of a given Hilbert space. Characterizing the state of a quantum system is crucial for fundamental studies in quantum mechanics as well as for manipulating and utilizing quantum systems for practical applications. One severe drawback of all currently available quantum metrology techniques is the need for a time-consuming characterization process, which scales very unfavorably with the dimensionality of the quantum system.

Here we describe a scan-free direct measurement approach that is capable of simultaneously measuring the entire state vector of a pure quantum system, consequently eliminating the need for scanning through each basis state. Our method involves a proper arrangement of weak [1,2] and strong measurements. Specifically, to measure the state vector of a quantum system in one Hilbert space $\mathscr{A}$, one first applies a weak measurement to the quantum system in one fixed state $\left|b_{0}\right\rangle$ of its complementary basis $\mathscr{B}$, and then performs the strong measurement directly in $\mathscr{A}$. Here, a weak measurement refers to the process of applying a weak operator $\hat{\pi}_{a}$ on the system with minimal perturbation such that the original quantum state $|\psi\rangle$ does not collapse fully until a second, conventional (also known as "strong") measurement is performed.

As an example, we here describe the procedure of measuring the transverse spatial state of a photon, which is a convenient high-dimensional quantum system for study, and which has a well-understood classical analogue as the transverse complex field profile of an optical beam. In order to measure the complex probability amplitude of a photon at a position state $x$, one first performs a weak projection measurement of the zero momentum state $p_{0}=0$, followed by a strong measurement of the position state. Through such a procedure, the measured weak value $\left\langle\pi_{p}\right\rangle_{x}^{\mathrm{w}}$ is given by

$$
\left\langle\pi_{p}\right\rangle_{x}^{\mathrm{w}}=\frac{\left\langle x \mid p_{0}\right\rangle\left\langle p_{0} \mid \psi\right\rangle}{\langle x \mid \psi\rangle}=\frac{v}{\psi(x)},
$$

where $v$ is a normalization constant, and $\psi(x)$ denote the state vector of the photon expressed in the position base.

One sees that the average result of such a measurement directly leads to the complex probability amplitude of the photon at position $x$. The main advantage of this approach is that the weak value $\left\langle\pi_{p}\right\rangle_{x}^{\mathrm{w}}$ at all positions can be measured simultaneously. This is because the strong measurement in $x$ can be performed on all position states at the same time through the use of an appropriate detector array. Thus, the need for a time-consuming scanning procedure is eliminated, and the entire state vector can be obtained with a single setting of the measurement apparatus.

Our experimental procedure is as follows. An ensemble of photons from a collimated laser beam with a fixed polarization state is first prepared using a phase-only spatial light modulator (SLM), which allows a high degree of control of the transverse spatial state $\psi(x)$ of the diffracted photons. The identically prepared photons pass through a $4 f$ imaging system. The weak measurement is first performed in the momentum space, i.e., the mutual focal plane of the two lenses, where a second phase-only SLM, in combination with two waveplates, is used to rotate the linear polarization of the photons in the zero-momentum state $|p\rangle=0$ through a small angle $\alpha$. Parallel strong measurements 
for all the position states are then simultaneously performed at the image plane of the $4 f$ system, during which the spatial polarization profile of the photons is measured. Formally, if one uses a two-dimensional vector, $\left[\begin{array}{ll}0 & 1\end{array}\right]^{\mathrm{T}}$, to denote the initial polarization state of the photons in the horizontal-vertical linear polarization basis, the complex probability amplitude of the photons in each position state $|x\rangle$ is given by

$$
\psi(x)=\langle x \mid \psi\rangle=\frac{v}{\left\langle\pi_{p}\right\rangle_{x}^{\mathrm{W}}}=\frac{v^{\prime}}{\left[\left\langle s_{\mathrm{f}}(x)\left|\hat{\sigma}_{1}\right| s_{\mathrm{f}}(x)\right\rangle-i\left\langle s_{\mathrm{f}}(x)\left|\hat{\sigma}_{2}\right| s_{\mathrm{f}}(x)\right\rangle\right]},
$$

where $\hat{\sigma}_{1}$ and $\hat{\sigma}_{2}$ are the first and second Pauli operators, respectively, $\left|s_{\mathrm{f}}(x)\right\rangle$ is the final polarization state at each position $x$ at the image plane, and $v$ and $v^{\prime}$ are constants determined through normalization.
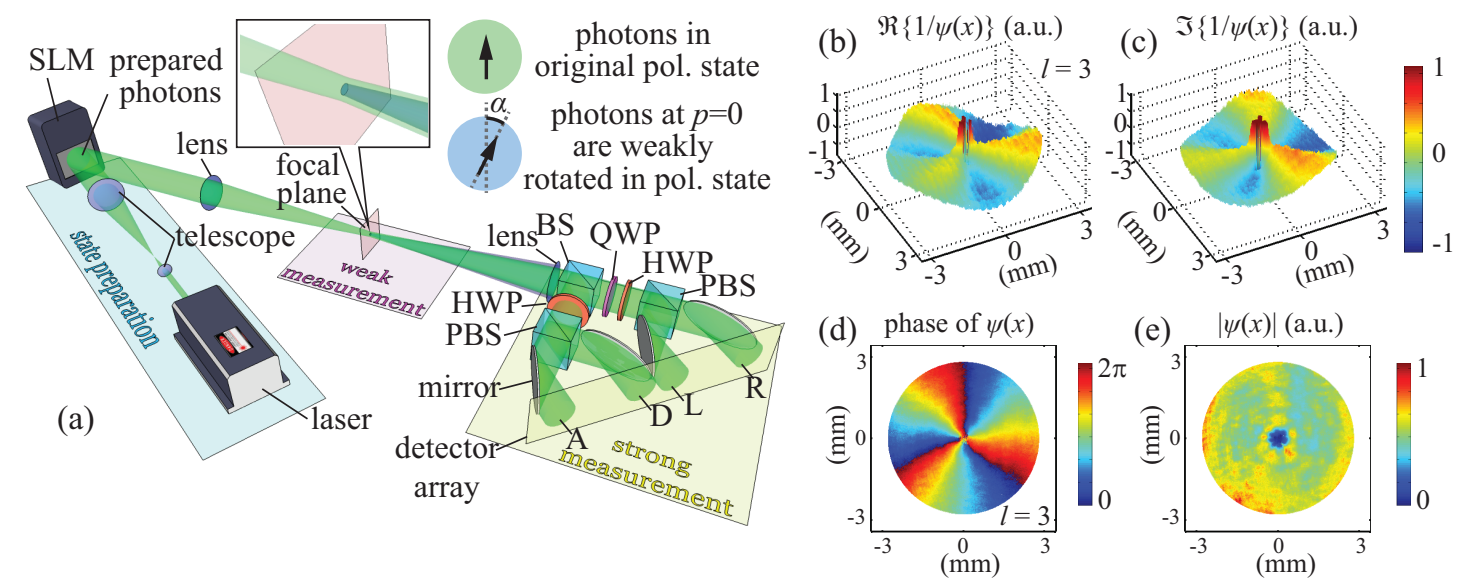

Fig. 1. (a) Schematic diagram of our scan-free direct measurement of the transverse wavefunction of photons; the measured real (b) and imaginary (c) parts of the two-dimensional weak values and the corresponding phase (c) and amplitude (d) profiles of the transverse spatial state of photons carrying orbital angular momentum (OAM) with quantum number $l=3$.

As an example of demonstration, we here characterize photons carrying orbital angular momentum (OAM) [3]. The real and imaginary parts of the measured weak value $\left\langle\pi_{p}\right\rangle_{x}^{\mathrm{w}}$ for photons with $l=3$ are plotted in Fig. 1(b) and (c), respectively. One sees that the magnitude of $\left|\left\langle\pi_{p}\right\rangle_{x}^{w}\right|$ becomes very large towards the center of the OAM beam, which is exactly expected due to the inverse relation between $\left\langle\pi_{p}\right\rangle_{x}^{\mathrm{w}}$ and the complex probability amplitude $\phi(x)$ of an OAM beam, which approaches zero towards the phase singularity at the center. The corresponding phase and amplitude of $|\psi(x)|$ is shown in Fig. 1(d) and (e), which accurately reveals the azimuthal phase structure and the central-null feature of the amplitude. Since one can have independent control of the complex probability amplitude of the photons at each pixel of the transverse space, the dimensionality of our measured state is approximately 1.2 million, which is determined by the spatial extent of the photons (approximately $7 \mathrm{~mm}$ in diameter) and the discrete nature of our detector array (with pixel size of $5.4 \mu \mathrm{m}^{2}$ ). The fidelity of the shown $l=3$ OAM mode in the spatial Hilbert space is calculated to be approximately 0.93 . Such a high fidelity of our result demonstrates that our direct measurement technique is indeed capable of measuring the complex-value quantum state vector with very high accuracy.

Our scan-free direct measurement approach opens up the possibility to characterizing high-dimensional quantum systems in real-time for which a state-by-state scanning process would become impractically time-consuming or even

infeasible. Moreover, our specific demonstration of measuring photons' transverse spatial state is also a promising new technology for classical wavefront sensing applications in fields as diverse as observational astronomy, free-space optical communication, and biomedical imaging.

\section{References}

1. Y. Aharonov, D. Z. Albert, and L. Vaidman, Phys. Rev. Lett. 60, 1351 (1988).

2. I. M. Duck, P. M. Stevenson, and E. C. G. Sudarshan, Phys. Rev. D 40, 2112 (1989).

3. A. M. Yao and M. J. Padgett, Adv. Opt. Photon. 3, 161 (2011). 